\title{
O prontuário do paciente e o dever legal e ético de registro dos profissionais da saúde: uma revisão literária
}

\author{
The patient's record and the legal and ethical duty to register health professionals: a \\ literature review
}
El registro del paciente y el deber legal y ético de registrar profesionales de la salud: una revisión de la literatura

Luis Eduardo Miami Gomes ${ }^{1}$, Juliana Teixeira Gomes ${ }^{2 *}$, Leandro Minitel Vidal de Negreiros ${ }^{1}$, Raquel Franco Leal ${ }^{1}$.

\section{RESUMO}

Objective: Descrever sobre a importancia dos registros no prontuário do paciente, tendo em vista a considerável relevância do dever legal e ético dos profissionais da saúde, além de destacar a importância deste documento. Revisão Bibliográfica: Em decorrência do valor legal e ético existente no prontuário do paciente, o emprego da correta forma de preenchimento deve ser um dos preceitos legais empregados na formação profissional. O prontuário do paciente é um documento individualizado e sigiloso, amparado pelos preceitos éticos e legais, e os registros ali contidos são extremamente relevantes ao paciente e aos profissionais envolvidos nessa assistência. Desse modo, o prontuário expressa a centralização das informações, da progressão do paciente e assim deve-se empregar a real importância das informações registradas, recebendo atenção dos profissionais, transgredindo o simples registro. Considerações finais: Os profissionais devem entender a seriedade no preenchimento do prontuário do paciente, inserindo na formação básica dos mesmos elementos subsidiadores ao correto preenchimento deste documento, e ao final torná-lo adequado e em conformidade aos preceitos éticos e legais de seu preenchimento, guarda e descarte.

Palavras-chaves: Prontuário, Registro, Confidencialidade.

\begin{abstract}
Objective: To describe the importance of the records in the patient's medical record, given the considerable relevance of the legal and ethical duty of health professionals. Bibliographic Review: Due to the legal and ethical value of the patient's medical record, following the correct way of filling it out must be one of the legal precepts used in professional training. The patient's medical record is an individualized and confidential document, supported by ethical and legal precepts, and the records contained therein are extremely relevant to the patient and the professionals involved in his or her treatment. In this way, the medical record shows thecentralization of information, the patient's progression, and the real purpose of the recorded information and should receivespecial attention from professionals more than as a simple record. Final considerations: Professionals must understand the seriousness in filling out the patient's medical record, inserting the basic training of the same elements that support the correct completion of this document, and in the end make it adequate and in accordance with the ethical and legal precepts of its filling, storage and disposal
\end{abstract}

Keywords: Medical record, Registration, Confidentiality.

${ }^{1}$ Faculdade de Ciências Médicas (FCM). Universidade Estadual de Campinas (Unicamp) Campinas, SP.

${ }^{2}$ Centro Universitário Salesiano de São Paulo (UNISAL), Americana, SP. *E-mail: ju.tgomes@hotmail.com

SUBMETIDO EM: 3/2020

ACEITO EM: 4/2020

PUBLICADO EM: 5/2020

REAS/EJCH | Vol.12(7) | e3615 | DOI: https://doi.org/10.25248/reas.e3615.2020 Página 1 de 8 


\section{RESUMEN}

Objetivo: Describir la relevancia de los registros en el registro del paciente, en vista de la considerable relevancia del deber legal y ético de los profesionales de la salud, además de resaltar la importancia de este documento. Revisión bibliográfica: debido al valor legal y ético existente en la historia clínica del paciente, el uso de la forma correcta de llenado debe ser uno de los preceptos legales utilizados en la formación profesional. El registro médico del paciente es un documento individualizado y confidencial, respaldado por preceptos éticos y legales, y los registros que contiene son extremadamente relevantes para el paciente y los profesionales involucrados en esta asistencia. De esta manera, el registro médico expresa la centralización de la información, la progresión del paciente y, por lo tanto, se debe utilizar la importancia real de la información registrada, recibiendo atención de profesionales y transgrediendo el registro simple. Consideraciones finales: Los profesionales deben comprender la seriedad en el llenado de la historia clínica del paciente, insertando en la capacitación básica los mismos elementos que respaldan la correcta realización de este documento, y al final hacerlo adecuado y cumplir con los preceptos éticos y legales de su llenado, almacenamiento y eliminación.

Palabras clave: Historia clínica, Registro, Confidencialidad.

\section{INTRODUÇÃO}

O registro das informações é uma das diversas atividades diárias dos profissionais da saúde, quando envolvidos na assistência ao paciente. Essas informações são aquelas passadas pelo próprio paciente, pelos responsáveis legais, ou ainda por ambos. Os elementos que constituem o registro são aqueles do qual o profissional alcançou, e aqueles que demonstrem a evolução da pessoa, além de apontar as medidas adotadas na terapia empregada. O prontuário é compreendido como uma coleção de elementosque são extraídos em diversas fontes, e assim armazenados, física ou eletronicamente (FERREIRA RG, et al., 2019).

Os elementos que subsidiam a elaboração do prontuário do paciente são os fornecidos em atendimento individualizado, sigilosamente e por esta razão os registros ali contidos só devem tornar-se público após seu expresso assentimento ou ainda de seu representante legal, ou por dever legal ou justa causa (MONTEIRO EKR, et al., 2019).

Importante destacarmos as definições e empregos das caracterizações do prontuário, que vem sendo empregado não exclusivamente como uma transcrição da anamnese do paciente, mas um conjunto de informações preconizadas, constituídas e referenciadas ao registro de cuidados dispensadosao paciente, como também uma reunião de documentos referentes à assistência (FRANÇA GV, 2013).

De acordo com Galvão MCB e Ricarte ILM (2012) trazem a definição de prontuário como sendo uma "coleção de informação relativa ao estado de saúde de um paciente, armazenada e transmitida em completa segurança e acessível ao paciente e a qualquer usuário autorizado".

Igualmente, segundo Coltri MV e Silva RHA (2019) trouxeram que o prontuário é o agrupamento de documentos elaborado e armazenado, e que tal documento possui valor ético legal, e que os elementos contidos neste documento possuem presunção de veracidade.

O prontuário é um espaço de centralização das informações, da progressão do paciente, e assim deve-se empregar a real importância das informações ali registradas, recebendo atenção destes profissionais, transgredindo o simples registro (da SILVA VA, et al., 2019).

Não se pode negar que o avanço da tecnologia vem interfaceando com o prontuário médico. Com esse avanço, os registros no prontuário tendem a progredir nessa esteira. Os registros impressos (prontuário físico) tendem a ficar em segundo plano, em substituição pelos meios eletrônicos. Embora essas modernizações dirigiram-se em sentido positivo, ainda existem dificuldades, bem como necessidades profissionais que devem ser sanadas (PALOMARES MLE e MARQUES IR, 2010; FRANCO MTG, et al., 2012). 
Inclusive essa informatização vem expressada na Lei 13.787 em dezembro de 2018, de considerável interesse e relevância sobre a digitalização e a utilização de sistemas informatizados para a guarda, o armazenamento e o manuseio de prontuário do paciente (BRASIL, 2018). Desse modo, eletronicamente ou ainda apresentado de forma física o registro em prontuário é um instrumento utilizado por diversos profissionais, a diversas finalidades, como a exemplo da extração de informações à produtividade, faturamento, evolução clínica e qualidade assistencial (POSSARI JF, 2007).

Logicamente o prontuário eletrônico apresenta algumas facilidades aos profissionais da saúde, que de forma sistematizada podem consultar registros anteriores com maior facilidade, e assim recordar de forma sistêmica os fatos e eventos clínicos ocorridos, sendo um importante veiculo de comunicação, uma interface entre profissionais (MONTEIRO EKR, 2019). Os processos de acreditação hospitalar também são outros processos que utilizam essa documentação legal, ponderando as anotações e registros como uma das circunstâncias avaliadas na qualidade assistencial e ainda na segurança do paciente (BRASIL, 2002).

Certamente, tendo em vista que os registros no prontuário serão inseridos por profissionais da qual prestaram assistência ao paciente, tais registros resguardam os profissionais ética e juridicamente por ser documento apto à defesa legal (BOMBARDA TB e JOAQUIM RHVT, 2019). Entretanto, evidenciou-se uma vulnerabilidade teórica na elaboraçãoem conexão procedimento da transcrição em prontuário e da certificação das atividades, conforme visto em estudo dos terapeutas ocupacionais (BOMBARDA TB e JOAQUIM RHVT, 2019).

Pelo valor legal empregado sobre esse documento, ignorar seu correto preenchimento é ir além de um desserviço, de ir a contramão, constitui infração grave atribuída e representada por cada conselho de classe. Sem dúvida um dos melhores caminhos ao correto preenchimento se dá pela capacitação pessoal e conscientização da grande relevância desse ato (BRANDÃO-SOUZA C, et al., 2019). Além disso, é respeitável padronizarmos os registros, levando em consideração sua importância, e julgando a potencialidade dessas informações para a continuidade assistencial e ainda para a constituição de um documento completo (GONDIN FBS, et al., 2019).

Diante do exposto, levando em conta e realçando a importância dos registros no prontuário, e pela considerável relevância nos diversos âmbitos desses registros, o presente estudo tem como objetivo divulgar e difundir através da literatura pertinente ao tema, sem, entretanto exaurir todo conteúdo, o prontuário e o dever dos profissionais em registrar as informações, além de destacar a relevância legal e ética deste documento.

\section{REVISÃO DE LITERATURA}

\section{A legislação brasileira}

Tendo em vista os aspectos legais envolvendo o prontuário, importante destacar que os registros contidos neles, referentes ao paciente são de sua pertença e declaram sobre sua vida privada e sobre sua intimidade. Assim, a confidencialidade das informações é uma prática na medicina desde Hipócrates (ANNAS GJ, 2003).

O prontuário é um agregado de documentos de relevância extremada que traduz o contato e a relação entre o paciente e a equipe de saúde (PRESTES JR, et al., 2007). Sendo o mesmo de extrema relevância, tanto em seu preenchimento, quanto documento hábil a resguardar os profissionais ética e juridicamente à defesa legal, a Lei 13.7871 de dezembro de 2018 que dispõe sobre a digitalização e a utilização de sistemas informatizados dispõe que para a guarda, o armazenamento e o manuseio de prontuário de paciente, em especial em seu artigo 6을 estabelece o prazo mínimo de 20 anos a partir da última escrita para guarda deste documento, sendo digitalizado ou ainda em papel (BRASIL, 2018). Relevante destacar que no parágrafo $3^{\circ}$ do artigo 6을 exibe destaque no descarte deste documento, que deverá resguardar a intimidade dos pacientes, o sigilo e a confidencialidade das informações (BRASIL, 2018).

Desse modo, expressamente previsto na Constituição Federal de 1988, no artigo 5으, inciso X a inviolabilidade da intimidade a vida privada, a honra e a imagem das pessoas, assegurado o direito à indenização pelo dano material ou moral decorrente de sua violação (BRASIL, 1988). Assim, o amparo Constitucional da inviolabilidade da intimidade e o sigilo das informações devem ser venerados acima de tudo. 
Certamente, as informações registradas no prontuário médico constituem como declarações verdadeiras em relação ao signatário. Essas informações vêm amparadas no Código de Processo Civil de 2015 em seu artigo 408, que "as declarações constantes do documento particular escrito e assinado ou somente assinado presumem-se verdadeiras em relação ao signatário" (BRASIL, 2015).

Temos ainda Lei n. 13.709, de 14 de agosto de 2018, que disciplina a proteção de dados pessoais e tem como fundamento dentre outros, o respeito à privacidade (artigo $2^{\circ}$, inciso I) (BRASIL, 2018). Ainda, a Lei prevê que toda "pessoa tem assegurada a titularidade de seus dados pessoais e garantidosos direitos fundamentais de liberdade, de intimidade e de privacidade" (BRASIL, 2018).

As informações registradas pelos profissionais que assistem o paciente durante uma de suas necessidades, seguramente considera-se o autor do documento, quando o fez e o assinou. Verifica-se que essa informação está também descrita no Código de Processo Civil de 2015 em seu artigo 410 e incisos (BRASIL, 2015).

Respeitável destacar que os profissionais podem ser penalizados por atos diversos daqueles considerados éticos e morais, e aqueles considerados ilícitos, como a falsificação do prontuário (falsificação de documento particular) ou ainda pela omissão em inserir ou fazer inserir declaração falsa ou diversa da que devia ser escrita, com o fim de prejudicar direito, criar obrigação ou alterar a verdade sobre fato juridicamente relevante, cometendo o crime de falsidade ideológica (BRASIL, 1940). Tendo em vista a discordância do paciente com os registros existentes em seu prontuário, competirá a ele mesmo o dever de comprovar a falta de fidedignidadeou a imprecisão do que consta (COLTRI MV e SILVA RHA, 2019).

Em que pese não distinguirmos e/ou apontarmos diferenças legais entre o prontuário eletrônico e físico em sua importância legal, de acordo com Gambi EMF (2013) em relação ao prontuário em papel, o prontuário eletrônico apresenta vantagens. O prontuário em papel apresenta incompreensibilidade dos registros, prejuízo dos elementos, pluralidade de pastas, problemas de pesquisas, falta de regulação e ajustamento, problemas de acesso, vulnerabilidade do papel e dificuldade deguarda.

Assim, de acordo com a legislação vigente, o prontuário é documento capaz de conter informações sigilosas e confidenciais, reconhecidamente importante à defesa de profissionais envolvidos a assistência ao paciente. Além disso, é documento de interesse do paciente as suas necessidades.

\section{Do código de ética}

Cumpre-nos esclarecer que os profissionais que tem o dever ético legal de preencher o prontuário do paciente, são aqueles que constituem parte de uma equipe multidisciplinar que prestam assistência ao paciente em suas diversas necessidades, e em seus diversos âmbitos.

Os profissionais que atuam no auxílio ao paciente, devem sob o fundamento aos seus respectivos códigos de ética, registrar as informações nos prontuários.

Assim, de acordo com as recomendações do guia de recomendações para o registro de enfermagem no prontuário do paciente, em decorrência da equipe enfermagem ser responsável por $50 \%$ dos registros, devido a grande quantidade de procedimentos que realizam, devem ser registrados de maneira legível e consideravelmente íntegra, de toda assistência e auxílio prestado (CONSELHO FEDERAL DE ENFERMAGEM, 2016).

O Conselho Federal de Enfermagem recomenda sobre a importância do preenchimento e sua finalidade, que além de compartilhar informações aos demais profissionais, proporciona informações de garantia de qualidade, bem como também evidências legais ao processo de auditoria, ensino e pesquisa (CONSELHO FEDERAL DE ENFERMAGEM, 2016).

Pela importância dos registros no prontuário e pelo adequado apontamento pelos profissionais envolvidos, o Conselho Federal de Fisioterapia e Terapia Ocupacional (COFFITO) disseminou sobre a obrigatoriedade do registro em prontuário pelos profissionais fisioterapeutas e terapeutas ocupacionais. Em concordância com a Resolução 415/2012 do COFFITO, define sobre plano assistencial, diagnóstico e prognóstico, além da identificação do profissional responsável por esse registro (CONSELHO FEDERAL DE FISIOTERAPIA E TERAPIA OCUPACIONAL, 2012). 
Ponderoso registrar que em que pese 0 artigo $6^{\circ}$, inciso I de a referida Resolução determinar expressamente sobre 0 tempo de preservação e sobre a conservação do prontuário do cliente/paciente/usuário ser de no mínimo cinco anos, podendo ser ampliado nos casos previstos em Lei, a contar do último registro (CONSELHO FEDERAL DE FISIOTERAPIA E TERAPIA OCUPACIONAL, 2012), ampliamente estabelece a Lei 13.787 de 2018 de prazo de 20 anos para guarda. Consequentemente, em vista da legislação vigente, e, em que pese a Resolução expressar que o prazo pode ser prorrogável nos casos previstos em Lei, entendemos que o tempo para guarda do prontuário deve ser aquele expressamente pela Lei 13.787 de 2018 de no mínimo 20 anos.

Em relação ao prontuário à equipe de nutricionistas, conforme a resolução do conselho federal de nutricionistas (CFN) considerando à implantação dos prontuários físicos ou eletrônicos um conjunto de elementos para uma comunicação e intercessão de comunicação partilhada entre os diversos profissionais da equipe de saúde, além das informações que diz respeito ao paciente (CONSELHO FEDERAL DE NUTRICIONISTAS, 2017).

Inclusive dispõe a Resolução do conselho federal de nutricionistas que o prontuário do paciente é um composto reunido e arranjado de documentos, elementos, sinais e imagens registrados, suscitados a partir de acontecimentos, eventos e circunstâncias sobre a saúde do paciente e ao auxílio a ele oferecido, de caráter legal, sigiloso e científico, que autoriza a interface dos componentes da equipe multidisciplinar, bem como a ininterrupção da assistência que é prestada ao paciente (CONSELHO FEDERAL DE NUTRICIONISTAS, 2017).

De conformidade com os demais códigos de éticas, o Conselho de Psicologia manifesta-se no sentido de tornar forçoso o apontamento documental sobre o atendimento prestado que não puder ser conservado majoritariamente sob o modo de prontuário psicológico, em decorrência das restrições do compartilhamento de informações (conselho federal de psicologia, 2009). Tanto quanto os demais códigos aqui expostos, o Conselho Federal de Psicologia também define o prontuário como caráter sigiloso e que este documento constitui um conjunto de elementos cujo objetivo é o registro prestado ao paciente, bem como a descrição, evolução e os procedimentos adotados (conselho federal de psicologia, 2009).

Sob o mesmo ponto de vista, o Conselho Federal de Medicina, por intermédio da Resolução ㄲo 1.638/2002 conceitua prontuário do paciente como documento único, cujo descritivo diz respeito aos sinais e sintomas, e imagens e percepções registradas a partir de um fato ou acontecimento, cujo componente primário é o atendimento prestado ao paciente. Ademais, deve ser de caráter sigiloso e que possibilite a comunicação entre os membros da equipe multiprofissional para a continuidade da assistência prestada (conselho federal de medicina, 2002).

O prontuário médico alcança grande significado entre o médico e o paciente, pois é neste documento que são registradas todas as assistências, abrangendo informações sobre seu estado de saúde, evolução, eventos pregressos, intercorrências e as medidas aplicáveis ao caso. Inclui ainda o relatório de alta, sendo este instrumento competente a definir a responsabilidade do profissional, de caráter fundamental para 0 seguimento clínico e documentação do paciente com relação à patologia e os procedimentos empregados (ARAÚJO ATM, et al., 2019).

Vale ressaltar a importância da qualidade com que as informações são registradas. Isso tem implicação direta na condução do caso, na avaliação da equipe multidisciplinar e na agilidade da obtenção das informações clínicas do paciente. Destaca-se ainda a relevância do registro da data, horário, nome e registro do profissional que está conduzindo o paciente (conselho federal de medicina, 2018). Ademais, temos ainda abarcado dentro desses conceitos e definições o caráter sigiloso que é protegido pelos princípios constitucionais que também garantem o direto à intimidade e privacidade dos indivíduos (SANTOS CSV, 2019).

Ainda de acordo com Araújo ATM, et al. (2019) este documento é um acúmulo de informações que tem direta relação com a saúde do paciente, e que este documento que foi produzido pelos profissionais envolvidos na assistência, pode ser um principal meio de defesa, permitindo através deste prontuário conferir as normas e procedimentos terapêuticos realizados. 
Todos os profissionais devem ter o entendimento acerca do prontuário do paciente, para que se possa manter a continuidade da assistência e sua legibilidade, pois em todos os códigos de éticas aqui descritos há menção a questão da legibilidade na escrita (MONTEIRO EKR, et al., 2019), Entendemos, portanto que o prontuário, contendo informações do paciente sob os cuidados que foram prestados deve ser único, exclusivo, e que devem estar disponíveis quando de sua solicitação.

\section{Das inadequações do prontuário}

Embora os preceitos legais e éticos, sob o amparo da importância de uma boa elaboração de um prontuário, sejam fundamentais, existem dados técnicos de que estes documentos não vêm sendo elaborados como determina os fundamentos.

Em que pese à importância deste documento, como suporte e auxílio em defesas técnicas e administrativas, em um estudo de Silva FG e Tavares-Neto J (2007) descrevem que os prontuários de hospitais escola, na generalidade é desanimador e necessita de urgentes medidas, até mesmo de uma intervenção na qualificação pessoal. Portanto, há apreensão com a boa propriedade do prontuário, que deve fazer parte do cotidiano e do desenvolvimento dos profissionais das instituições de saúde, principalmente as universitárias, dos quais a formação deve ser instituída de forma integral e de abrangida categoria, a fim de aprovar as pretensões de uma coletividade cada vez mais rigorosa e mais atenta aos detalhes (PRESTES JR, et al., 2007).

A representatividade nos registros, tanto eletrônico quanto físico, é concordante no que tange sua importância e foram apresentados divergências no que refere a infraestrutura ou ainda pela inexistência de registro informatizado ao prontuário eletrônico. Descreve-se ainda que a dificuldade encontrada em alguns casos ao exercício profissional foi em decorrência da ausência de habilidade tecnológica ou ainda pela limitação estrutural apresentada (PEREIRA APN, et al., 2019).

Inclusive, em algumas análises de prontuários puderam expressar a qualidade dos registros e da assistência prestada ao paciente. Entretanto, quando se busca uma avaliação mais objetiva, o registro demonstra a necessidade de um maior refinamento e de um método de educação mais apurado, centrado na importância e gerenciamento dos cuidados (CARVALHO RS, et al., 2019).

Em recente estudo sobre a análise dos prontuários, Garritano CR, et al. (2020) observaram que na análise dos itens havia uma insuficiência na elaboração dos prontuários com vários itens inacabados e/ou inexistentes. Além disso, do total dessa amostra analisada, $32 \%$ dos prontuários foram considerados inadequados e os exames complementares não foram encontrados em $44,5 \%$ da amostra.

Entretanto, o Conselho Federal de Medicina vem desempenhando atividade de refinamento quanto à forma correta de preenchimento dos documentos e elaboração do prontuário com a implantação da Comissão de Revisão de Prontuário, através da Resolução do Conselho Federal de Medicina no 1.638 de 9 de agosto de 2002 (PRESTES JR, et al., 2007).

Não obstante os trabalhos cujo objetivo é a melhora da elaboração dos prontuários, temos que destacar que existem alguns fatores que possam interferir nesse registro. $O$ fator carga de trabalho é um grande influenciador na pratica diária da equipe multiprofissional. Por conseguinte, surge também a necessidade de ações e intervenções educativas e até mesmo de conteúdo revisional durante a formação destes profissionais (BOSCO PS, et al., 2019).

Claro que a insuficiência de profissionais tem impacto negativo na elaboração do prontuário, e há uma priorização e distribuição de atividades, implicando positiva ou negativamente sobre os cuidados (BOSCO PS, et al., 2019).

Acresce que para a melhora da qualidade dos registros em prontuários, deve haver capacitação e padronização destes registros nos serviços de saúde. Importante ainda que essa capacitação possa atingir a formação acadêmica, e que esta atuação interfira diretamente na prática, e que futuramente estes profissionais atuem registrando a continuidade da assistência de forma segura e adequada (GONDIN FBS, et al., 2019). 
Numa análise retrospectiva de prontuários de registros de violência doméstica, observou-se um crescente número de registro do problema dos pacientes, mas, mesmo assim, houve insuficiente descrição sobre a informação de como tal problema foi trabalhado nesses serviços (DARMAU EC e TERRA MF, 2019).

Conquanto o sigilo profissional seja a base na relação profissional paciente, as medidas interventivas devem ser impostas, o ensino na graduação deve fornecer elementos para aumentar o conhecimento sobre sigilo profissional (LUTZ KT, 2019).

Assim sendo, a capacidade técnico-científica da equipe de saúde é indispensável para registros completos. Reflete-se, pois sobre a obrigação de instituições e órgãos de classerealçar e promovernotas que auxiliem na capacitação do profissional para que não haja comprometimento na garantiada particularidade da assistência prestada e segura ao paciente (FERREIRA LL, et al., 2020).

Os resultados encontrados nos estudos revelam-se contrariamente aos códigos de éticas dos profissionais de saúde, pois a incompletude e a falta de registros nos prontuários estão divergentes dos preceitos éticos das profissões, além dos fundamentos legais expostos.

\section{CONSIDERAÇÕES FINAIS}

O presente estudo corroborou com a importância do registro dos profissionais de saúde que prestam assistência ao paciente. A seriedade do registro deve ser entendida não apenas como um indicador da qualidade da assistência ou ainda um método a ser empregado nas auditorias em saúde, mas um documento de comunicação do paciente com a equipe de atendimento no seguimento do paciente. Além disso, o prontuário é um documento, um testemunho de uma assistência prestada, capaz de ser um elemento hábil para a prestação de defesa técnica (judicial e administrativa) dos profissionais, respeitando claro o sigilo existente nos respectivos códigos de éticas, e em especial na legislação Constitucional vigente. Evidente, pois que há necessidade de ampliação e extensão da importância e da valiosidade dos registros no prontuário do paciente, pelos profissionais de saúde.

\section{REFERÊNCIAS}

1. ANNAS GJ. HIPPAA Regulations - A new era of medical-record privacy? N. Engl. J Med 2003; 348: 148690.

2. ARAÚJO ATM, et al. O sigilo do prontuário médico como um direito essencial do paciente: uma análise a partir das normativas do Conselho Federal de Medicina. Cadernos Ibero-Americanos de Direito Sanitário. 2019 jan./mar.; 8(1): 95-109.

3. BRASIL. CP. Decreto-Lei № 2.848, de 7 de dezembro de 1940. Diário Oficial da União, Rio de Janeiro, 1ํ de janeiro de 1942.

4. BRASIL C (1988). Constituição da República Federativa do Brasil. Brasília, DF: Senado Federal.

5. BRASIL. Ministério da Saúde. Secretaria de Assistência à Saúde. Manual brasileiro de acreditação hospitalar. Brasília, 2002.

6. BRASIL. Lei no 13.105, de 16 de março de 2015. Institui o Código de Processo Civil. Diário Oficial da União, Brasília, DF, 17 março 2015.

7. BRASIL. Lei no 13.787, de 27 de dezembro de 2018. Dispõe sobre a digitalização e a utilização de sistemas informatizados para a guarda, o armazenamento e o manuseio de prontuário de paciente.

8. BRASIL. Lei n. 13.709, de 14 de agosto de 2018. Lei Geral de Proteção de Dados Pessoais (LGPD).

9. BRANDAO-SOUZA C, et al. Completude dos prontuários de idosas com câncer de mama: estudo de tendência. Acta paul. enferm., São Paulo, 2019; 32(4): 416-424.

10. BOMBARDA TB; JOAQUIM RHVT. O ensino do registro em prontuário no campo da terapia ocupacional hospitalar. Cad. Bras. Ter. Ocup., São Carlos, 2019; 27(3): 593-601.

11. BOSCO PS, et al. Registros de enfermagem e suas implicações para a qualidade do cuidado. São Paulo: Revista Recien, 2019; 9(26): 3-10.

12. CARVALHO RS, et al. Avaliação qualitativa dos registros de enfermagem como estratégia educativa e segurança para o registro do paciente. Semana de Enfermagem, 2019.

13. COLTRI MV; SILVA RHA. Prontuário do paciente: comentários à lei no 13787/2018. Rev. Bras. Odontol. Leg. RBOL, 2019; 6(2): 89-105.

14. CONSELHO FEDERAL DE ENFERMAGEM (COFEN). Guia de Recomendações para o Registro de Enfermagem no prontuário do paciente e outros documentos de enfermagem. Brasília (DF): COFEN; 2016. 
15. CONSELHO FEDERAL DE FISIOTERAPIA E TERAPIA OCUPACIONAL. RESOLUÇÃO № 415/2012. Dispõe sobre a obrigatoriedade do registro em prontuário pelo terapeuta ocupacional, da guarda e do seu descarte e dá e dá outras providência; 2012.

16. CONSELHO FEDERAL DE NUTRICIONISTAS (CFN). Código de Ética do Nutricionista. Resolução CFN № 594, DE 17 DE DEZEMBRO DE 2017. Dispõe sobre o registro das informações clínicas e administrativas do paciente, a cargo do nutricionista, relativas à assistência nutricional, em prontuário físico (papel) ou eletrônico do paciente; 2017.

17. conselho federal de medicina (CFM). Código de Ética Médica. Resolução CFM n 2.217 de 27 de setembro de 2018. Brasília. Publicada no D.O.U. de 01 de novembro de 2018, Seção I, p. 179.

18. conselho federal de medicina (CFM). Define prontuário médico e torna obrigatória a criação da Comissão de Revisão de Prontuários nas instituições de saúde. Resolução CFM no 1638, de 9 de agosto de 2002.

19. conselho federal de psicologia. Resolução CFP no 001/2009. Dispõe sobre a obrigatoriedade do registro documental decorrente da prestação de serviços psicológicos, 2009.

20. da SILVA VA, et al. Auditoria da qualidade dos registros de enfermagem em prontuários em um hospital universitário. Enfermagem em Foco, 2019; 10(3).

21. DARMAU EC, TERRA MF. Registros de violência doméstica de gênero nos prontuários família na Atenção Primária à Saúde. Arq. Med. Hosp. Fac. Cienc. Med Santa Casa São Paulo, São Paulo, 2019; 64(1): 35-39.

22. FERREIRA RG, et al. Prontuário médico: uma revisão bibliográfica. Revista Bioética Cremego, 2019; 01(1): 2-8.

23. FERREIRA LL, et al. Análise dos registros de técnicos de enfermagem e enfermeiros em prontuários. Rev. Bras. Enferm., Brasília, 2020; 73(2): e20180542.

24. FRANCO MTG, et al. Avaliação dos registros de enfermeiros em prontuários de pacientes internados em unidade de clínica médica. Acta paul. Enferm, 2012; 25(2); 163-170.

25. FRANÇA GV. Direito médico. 11. ed. rev., atual. e ampl. Rio de Janeiro: Forense; 2013.

26. GAMBI EMF. A transição do prontuário do paciente em suporte papel para o prontuário eletrônico do paciente e seu impacto para os profissionais de um arquivo de instituição de saúde. Rev. Eletr. de Com. Inf. Inov. Saúde, 2013; 7(2).

27. GALVÃO MCB e RICARTE ILM. Prontuário do paciente. Rio de Janeiro: Guanabara Koogan; 2012.

28. GARRITANO CRO, et al . Avaliação do Prontuário Médico de um Hospital Universitário. Rev. bras. educ. med., Brasília, 2020; 44(1): e009.

29. GONDIM FBS, et al. A importância dos registros das infecções relacionadas à assistência à saúde no sumário de alta. Connectionline n.21 - 2019.

30. LUTZ KT, et al. Sigilo profissional: conhecimento de alunos de medicina e médicos. Rev. Bioét., Brasília, 2019; 27(3): 471-481.

31. MONTEIRO EKR, et al. Prontuário eletrônico como ferramenta da gestão do cuidado: uma proposta para atualização. Revista de Saúde Dom Alberto, 2019; 4(1): 77-90.

32. PALOMARES MLE e MARQUES IR. Contribuições dos sistemas computacionais na implantação da sistematização da assistência de enfermagem. Journal of Health Informatics, 2010; 2(3); 78-82.

33. PEREIRA APN, et al. Representações sociais de enfermeiros da atenção primária sobre registros de enfermagem em prontuários. Enferm Bras, 2019; 18(6): 759-66.

34. POSSARI JF. Prontuário do paciente. In: POSSARI, J. F. Prontuário do paciente e os registros de enfermagem. São Paulo: Látria, 2007; 17-34.

35. PRESTES JR, et al. Prontuário médico e suas implicações médico-legais na rotina do colo-proctologista. Rev bras. colo-proctol., Rio de Janeiro, 2007; 27(2): 154-157.

36. SANTOS CSV. Análise da quebra do sigilo médico profissional nos casos de aborto dentro do direito brasileiro. JiParaná: Centro Universitário São Lucas, 2019.

37. SILVA FG e TAVARES-NETO J. Avaliação dos prontuários médicos de hospitais de ensino do Brasil. Rev. bras. educ. med., Rio de Janeiro, 2007; 31(2): 113-126. 